\title{
Pre-vascularization of bone tissue-engineered constructs
}

\author{
Meadhbh Aín Brennan ${ }^{1}$, Jean-Michel Davaine ${ }^{1,2^{*}}$ and Pierre Layrolle \\ See related research by Pedersen et al., http://stemcellres.com/content/4/3/52
}

\begin{abstract}
Vascularization remains one of the primary obstacles in the repair of bone defects. In the previous issue of Stem Cell Research \& Therapy, Pedersen and colleagues show that co-immobilization of endothelial cells and mesenchymal stem cells in a tissue-engineered construct can achieve functional microvascular networks in vivo. These very interesting findings, together with other state-of-the-art research in this field, are presented in this commentary. They highlight the vital role of mesenchymal stem cells as supporting cells to nascent blood vessels. The development of pre-vascularized implants by using clinically relevant cell sources, which could lead to rapid integration into the host tissue, would be of immense interest.
\end{abstract}

In tissue engineering, the repair of critical-sized bone defects by using cells seeded onto biomaterial scaffolds remains a major challenge. It is well documented that the lack of vascularization within an engineered construct is the most important inhibiting factor for healing large bone defects. Proximity to a vascular network is necessary for survival of cells, adequate delivery of nutrients and oxygen, and removal of waste products. Consequently, cell death often occurs in the center of implants prior to vessel in-growth from the surrounding host tissue. Therefore, strategies that address the means of improving angiogenesis of bone tissue-engineered implants are vital. The present article is a commentary on the original research article by Pedersen and colleagues [1], which appeared in the previous issue of Stem Cell Research \& Therapy

\footnotetext{
* Correspondence: davainej@yahoo.fr

${ }^{1}$ Laboratory of the Pathophysiology of Bone Resorption, INSERM UMR957,

Faculty of Medicine, University of Nantes, 1 rue Gaston Veil, Nantes cedex 1 44035, France

${ }^{2}$ Vascular Surgery Department, Nantes University Hospital, Hôpital G et R Laënnec, Boulevard Professeur Jacques Monod, St-Herblain 44800, France
}

and which is of utmost interest in this perspective. The authors created three-dimensional microvascular networks under dynamic culture conditions in vitro prior to implantation in vivo. The study consisted of fabricating porous copolymer scaffolds of poly(LLA-co-DXO) that were seeded either with human bone marrow-derived mesenchymal stem cells (hMSCs) alone or in unison with human umbilical vein endothelial cells (hUVECs). These three-dimensional constructs were maintained for 1 week in vitro prior to implantation subcutaneously in mice. Importantly, the authors showed that endothelial microvascular networks were observed after 1 week of in vitro culture and sustained after in vivo implantation within hUVEC/hMSC constructs. Moreover, incorporation of the implanted microvascular network with the host vasculature was observed after only 1 week of implantation in vivo [1].

The addition of hUVECs to mesenchymal stem cells (MSCs) influenced both angiogenic and osteogenic gene expression profiles [1]. However, the crosstalk molecules between these two types of cells as well as privileged molecular pathways toward gene expression remain unclear. Upregulated human vascular endothelial growth factor (VEGF) expression was observed in hUVEC/hMSC co-cultures compared with MSC monocultures after 3 weeks in vivo. Previous studies have shown that MSCs and osteoblasts produce VEGF and other paracrine signals that increase the survival and growth of endothelial cells $[2,3]$ and that mechanical loading of MSCs further enhances their paracrine pro-angiogenic properties [3]. Interestingly, Pedersen and colleagues [1] demonstrated that MSCs could support blood vessel formation in vivo by differentiating into perivascular cells, in agreement with previous findings [4]. Nevertheless, the perivascular transformation of MSCs was not enhanced by implanted hUVECs [1].

It has previously been shown that hUVECs enhanced the osteogenic differentiation of MSCs in vitro [5-7] and in vivo [5]. Grellier and colleagues [5] co-cultured 
hUVECs and hMSCs inside RGD (arginine-glycineaspartic acid)-grafted alginate microspheres under dynamic conditions in vitro prior to implantation in a bone defect in vivo. Osteogenic gene expression, as well as mineralization in vitro and in vivo, was upregulated in cocultures compared with monocultures of hMSCs [5]. Bidarra and colleagues [6] revealed that hUVECs, in addition to modulating the osteogenic differentiation of hMSCs, increase the proliferation rate of hMSCs. In another study, hUVECs enhanced the osteogenic differentiation of MSCs in a three-dimensional co-culture in vitro model through upregulation of bone morphogenetic expression [7]. Furthermore, the presence of hUVECs inhibited the adipogenic differentiation of MSCs by enhancing Wnt signaling [7]. Nevertheless, unlike in these previous studies, no difference in osteogenic gene expression, alkaline phosphatase, or alizarin red staining was observed in co-culture constructs compared with monoculture of hMSCs after in vivo implantation of threedimensional constructs [1].

Most studies aiming to pre-vascularize implants for bone repair use hUVECs; however, these are macrovascular cells and therefore it would be interesting to assess the influence of microvascular cells, originating from capillaries, on MSC activity. Furthermore, it is known that macro- and microendothelial matrix components differ significantly and thus affect MSCs in different manners [8]. In this regard, Santos and colleagues [9] co-cultured human dermal microvascular endothelial cells with human osteoblasts (hOBs) in three dimensions. The authors achieved formation of a network of microcapillary-like structures, and $\mathrm{hOB}$ in co-culture significantly upregulated type I collagen and secreted higher amounts of VEGF compared with hOBs in monoculture [9]. A further microvascular cell type of interest would be pericyte cells. Indeed, in the setting of atherosclerosis, bone-like vascular calcifications co-localize with intraplaque angiogenesis, and microvascular pericytes are suspected to play a critical role in both mineralization and angiogenesis in atherosclerotic lesions [10].

Achieving the optimal culture conditions for prevascularized implants is imperative, and cell types, media components, cell densities, temporal variations in adding cells to co-culture, and dynamic loading are among the most important factors to consider. An important study by Correia and colleagues [11] demonstrated that, in tissue-engineered constructs, vasculogenesis should be induced prior to osteogenesis in vitro in order to obtain functional vasculature and osteoid formation following implantation in vivo.

For clinical applications of tissue engineering, vascular endothelial cells are inappropriate as they are not easily accessible and cannot be attained in sufficient quantities. Therefore, strategies to pre-vascularize implants by other means are under investigation. It has been shown that progenitor-derived endothelial cells enhance the differentiation of hMSCs to the same degree as hUVECs [12]; and Moioli and colleagues [13] have shown promising results for co-culture of hematopoietic and mesenchymal stem/ progenitor cells in enhancing vascularization and osteoid formation in tissue-engineered constructs in vivo. The differentiation of MSCs into endothelial and pericyte-like cells has previously been shown in two-dimensional in vitro studies $[14,15]$. However, landmark studies showed that MSCs alone are not entirely adequate for attaining fully vascularized implants [4,16]. Duffy and colleagues [16] achieved capillary-like networks in vitro by using MSCs cultured with endothelial supplements within collagen-glycosaminoglycan scaffolds; however, vital endothelial cell markers were not present. Au and colleagues [4] demonstrated the need for both endothelial cells and MSCs to achieve functional blood vessels in vivo. A potential mechanism for pre-vascularized constructs,
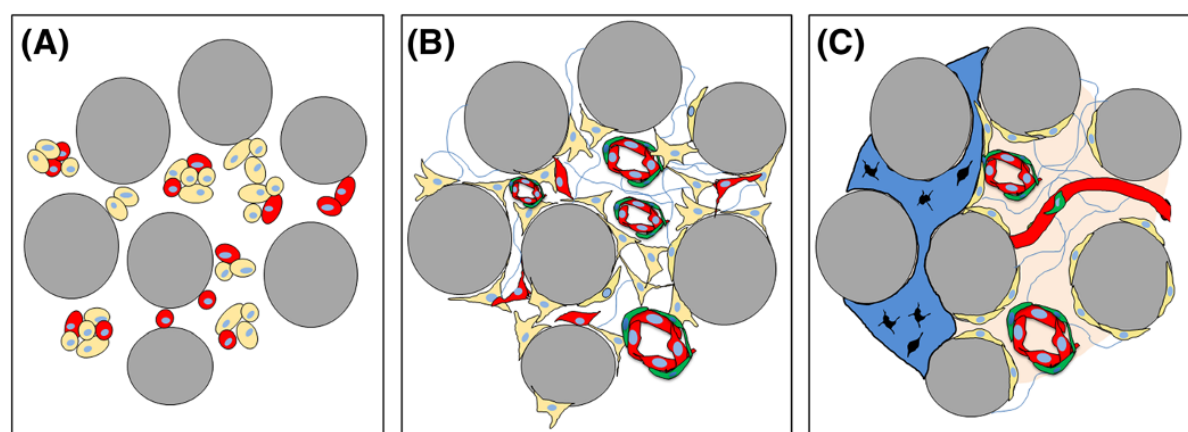

Figure 1 Schematic of possible mechanism of pre-vacularized implants for bone repair. (A) Endothelial cells (red) and mesenchymal stem cells (MSCs) (yellow) are co-immobilized within a biomaterial scaffold (grey) in vitro. (B) Cells attach to the surface of biomaterial. Endothelial progenitor cells form a microvascular network, and some MSCs which have been exposed to endothelial growth supplements differentiate into perivascular cells (green). Other MSCs exposed to osteogenic supplement differentiate toward osteoblast cells. (C) After implantation in vivo, perivascular cells act to support blood vessels, the microvascular network fuses with host vasculature, osteoblasts line the surface of biomaterials, and new bone is formed with embedded osteocytes (black). 
which encompasses many of the important features of the research articles highlighted in this commentary, is presented in Figure 1.

In conclusion, together these studies show that endothelial cells and MSCs enjoy a symbiotic relationship whereby they enhance osteogenic differentiation and angiogenesis, respectively. A crucial role of MSCs in attaining functional vascularized engineered scaffolds, as supporting cells to nascent blood vessels, has been demonstrated. Future work should explore the potential of three-dimensional pre-vascularized constructs by using more easily attainable, clinically relevant cell sources. Such a strategy, which may achieve rapid anastomosis in vivo and thereby greatly enhance bone repair and carry the possibility of patientspecific constructs, would be of the utmost interest to the bone tissue-engineering field.

\section{Abbreviations}

hMSC: Human bone marrow-derived mesenchymal stem cell; hOB: Human osteoblast; hUVEC: Human umbilical vein endothelial cell; MSC: Mesenchymal stem cell; VEGF: Vascular endothelial growth factor.

\section{Competing interests}

The authors declare that they have no competing interests.

Published: 14 August 2013

\section{References}

1. Pedersen TO, Blois AL, Xing Z, Xue Y, Sun Y, Finne-Wistrand A, Akslen LA, Lorens JB, Leknes KN, Fristad I, Mustafa K: Endothelial microvascular networks affect gene expression profiles and osteogenic potential of tissue-engineered constructs. Stem Cell Res Ther 2013, 4:52.

2. Grellier M, Bordenave L, Amédée J: Cell-to-cell communication between osteogenic and endothelial lineages: implications for tissue engineering. Trends Biotechnol 2009, 27:562-571.

3. Kasper G, Dankert N, Tuischer J, Hoeft M, Gaber T, Glaeser JD, Zander D, Tschirschmann M, Thompson M, Matziolis G, Duda GN: Mesenchymal stem cells regulate angiogenesis according to their mechanical environment. Stem Cells 2007, 25:903-910

4. Au P, Tam J, Fukumura D, Jain RK: Bone marrow-derived mesenchymal stem cells facilitate engineering of long-lasting functional vasculature. Blood 2008, 111:4551-4558

5. Grellier M, Granja PL, Fricain J-C, Bidarra SJ, Renard M, Bareille R, Bourget C, Amédée J, Barbosa MA: The effect of the co-immobilization of human osteoprogenitors and endothelial cells within alginate microspheres on mineralization in a bone defect. Biomaterials 2009, 30:3271-3278.

6. Bidarra SJ, Barrias CC, Barbosa MA, Soares R, Amédée J, Granja PL: Phenotypic and proliferative modulation of human mesenchymal stem cells via crosstalk with endothelial cells. Stem Cell Res 2011, 7:186-197.

7. Saleh FA, Whyte M, Genever PG: Effects of endothelial cells on human mesenchymal stem cell activity in a three-dimensional in vitro model. Eur Cell Mater 2011, 22:242-257. discussion 257.

8. Lozito TP, Kuo CK, Taboas JM, Tuan RS: Human mesenchymal stem cells express vascular cell phenotypes upon interaction with endothelial cell matrix. J Cell Biochem 2009, 107:714-722.

9. Santos MI, Unger RE, Sousa RA, Reis RL, Kirkpatrick CJ: Crosstalk between osteoblasts and endothelial cells co-cultured on a polycaprolactone-starch scaffold and the in vitro development of vascularization. Biomaterials 2009, 30:4407-4415.

10. Collett GD, Canfield AE: Angiogenesis and pericytes in the initiation of ectopic calcification. Circ Res 2005, 96:930-938.

11. Correia C, Grayson WL, Park M, Hutton D, Zhou B, Guo XE, Niklason L, Sousa RA, Reis RL, Vunjak-Novakovic G: In vitro model of vascularized bone: synergizing vascular development and osteogenesis. PLoS One 2011, 6:e28352.
12. Thébaud NB, Siadous R, Bareille R, Remy M, Daculsi R, Amédée J, Bordenave L: Whatever their differentiation status, human progenitor derived - or mature endothelial cells induce osteoblastic differentiation of bone marrow stromal cells. J Tissue Eng Regen Med 2012, 6:e51-e60.

13. Moioli EK, Clark PA, Chen M, Dennis JE, Erickson HP, Gerson SL, Mao JJ: Synergistic actions of hematopoietic and mesenchymal stem/progenitor cells in vascularizing bioengineered tissues. PLoS One 2008, 3:e3922.

14. Oswald J, Boxberger S, Jørgensen B, Feldmann S, Ehninger G, Bornhäuser M, Werner C: Mesenchymal stem cells can be differentiated into endothelial cells in vitro. Stem Cells 2004, 22:377-384.

15. Bouacida A, Rosset P, Trichet V, Guilloton F, Espagnolle N, Cordonier T, Heymann D, Layrolle P, Sensébé L, Deschaseaux F: Pericyte-like progenitors show high immaturity and engraftment potential as compared with mesenchymal stem cells. PLoS One 2012, 7:e48648.

16. Duffy GP, McFadden TM, Byrne EM, Gill S-L, Farrell E, O'Brien FJ: Towards in vitro vascularisation of collagen-GAG scaffolds. Eur Cell Mater 2011, 21:15-30.

doi:10.1186/scrt307

Cite this article as: Brennan et al:: Pre-vascularization of bone tissueengineered constructs. Stem Cell Research \& Therapy 2013 4:96. 